\title{
INTERAÇÕES UNIVERSIDADE-EMPRESA: UM ESTUDO EXPLORATÓRIO SOBRE AS EMPRESAS DO SETOR FARMACÊUTICO.
}

\author{
Nathalia Guimarães Alves ${ }^{1}$ \\ Marco Antônio Vargas ${ }^{2}$ \\ Jorge Nogueira Paiva Britto ${ }^{3}$
}

\begin{abstract}
Resumo
O artigo consiste em um estudo exploratório sobre perfil das empresas que compõem o setor farmacêutico e biofarmacêutico nacional sob um enfoque de interações universidade-empresa. O estudo concentra-se na análise empresas dedicadas à fabricação de produtos farmacêuticos e farmoquímicos e empresas dedicadas à pesquisa de desenvolvimento científico que mantiveram relacionamentos com os grupos de pesquisa cadastrados no Conselho Nacional de Desenvolvimento Científico e Tecnológico (CNPq) segundo informações coletadas no Censo 2010. Os dados permitem abordar questões como o número de empresas envolvidas em interações universidade-empresa, os tipos de relacionamentos estabelecidos, o tamanho, a dispersão geográfica e a natureza jurídica das empresas catalogadas sob os ramos de atividade selecionados e envolvidas com os grupos de pesquisa do CNPq.
\end{abstract}

\begin{abstract}
The paper addresses the profile of the companies that integrate the national pharmaceutical and biopharmaceutical industry based on a university-industry relationships scope. The study focuses on the analysis of companies dedicated to pharmaceuticals and pharma-chemicals manufactory and companies dedicated to scientific research and development that held relationships with research groups registered in the Brazilian National Council for Scientific and Technological Development (CNPq) according to data collected in the census 2010. The data allows us to discuss topics such as the number of companies involved in university-industry interactions, the types of relationships stablished, the size, the geographic dispersion and the legal nature of companies cataloged under selected activity fields which held relationships with the CNPq research groups.
\end{abstract}

Palavras chave: Indústria farmacêutica, biotecnologia, empresas de base tecnológica, interações universidade-empresa.

Código JEL L0, L65, O30, O31, O54.

ABEIN Área 6

\footnotetext{
${ }^{1}$ Aluna de mestrado do Programa de Pós-graduação em Economia da Universidade Federal Fluminense (PPGE-UFF)

2 Professor do Instituto de Economia da Universidade Federal Fluminense (UFF)

${ }^{3}$ Professor do Instituto de Economia da Universidade Federal Fluminense (UFF)
} 


\section{Introdução}

A indústria farmacêutica mundial é conhecida na literatura como uma indústria baseada em ciência, na qual os conhecimentos gerados na esfera acadêmica e as relações entre universidades e empresas são relevantes para a geração de novos compostos e drogas terapêuticas. O surgimento da biotecnologia moderna, nas décadas de 1970 e 1980, representou a ruptura do paradigma tecnológico no qual se baseava a indústria farmacêutica mundial. O choque tecnológico impactou todos os estágios da cadeia de pesquisa, desenvolvimento e inovação (PD\&I) e originou profundas mudanças na organização das firmas e nas interações entre os atores do sistema setorial de inovação farmacêutica no mundo. O processo resultou na composição de um sistema setorial de inovação no qual a interação entre agentes heterogêneos, tais como universidades, empresas farmacêuticas e empresas dedicadas à biotecnologia são centrais para a geração de inovações.

No Brasil, a estrutura da indústria farmacêutica envolve a presença de cinco agentes fundamentais: as empresas farmacêuticas nacionais, as grandes empresas farmacêuticas multinacionais instaladas no país, as firmas dedicadas à biotecnologia para saúde humana, os laboratórios públicos de pesquisa e produção de fármacos e medicamentos e as universidades e centros de pesquisa acadêmica. Dois fatores foram essenciais para o molde da estrutura da indústria farmacêutica no país. Por um lado, a existência de um modelo de saúde pautado pela universalidade e pela presença ativa do Estado na provisão de serviços de saúde, através da organização do Sistema Único de Saúde (SUS) e do Complexo Econômico-Industrial da Saúde (CEIS), resultaram na elevada participação de laboratórios oficiais de pesquisa e produção de fármacos e biofármacos no âmbito do sistema farmacêutico nacional e na importância estratégica da nacionalização da produção de biofármacos em virtude de seu grande peso relativo nos déficits históricos da balança comercial do CEIS. Por outro lado, o fortalecimento das empresas farmacêuticas nacionais através da estratégia de produção local de medicamentos genéricos, regulada pela lei $n^{\circ}$ 9789/1999 ${ }^{4}$, implicou em um aumento da participação das empresas nacionais no mercado farmacêutico brasileiro e representou um ponto de inflexão no processo de aprendizagem e construção de competências das empresas farmacêuticas locais. Contudo, apesar dos avanços alcançados pelas empresas farmacêuticas brasileiras desde a implementação da lei dos genérico de 1999, a indústria farmacêutica nacional ainda apresenta gargalos estruturais na condução de processos internos de PD\&I e na inserção no segmento de drogas terapêuticas produzidas por rotas biotecnológicas.

Neste contexto, o artigo visa contribuir para a literatura especializada através de uma abordagem diferenciada da questão das interações entre instituições acadêmicas brasileiras e empresas que compõem o sistema farmacêutico nacional. A análise está focada nas empresas dedicadas à produção de produtos farmacêuticos ou farmoquímicos e empresas envolvidas na condução de pesquisa e desenvolvimento científico. Para a condução da pesquisa foram selecionados dados de interações entre universidades e empresas disponíveis no Diretório Nacional dos Grupos de Pesquisa do Conselho Nacional de Desenvolvimento Científico e Tecnológico (CNPq) referentes ao censo 2010. Os dados do diretório dos grupos de pesquisa do CNPq permitem explorar o perfil das empresas e instituições que interagem com universidade, conforme: tamanho da empresa, dispersão geográfica, a natureza jurídica das empresas e tipos de relacionamentos estabelecidos com os grupos de pesquisa cadastrados no CNPq. Para a atualização dos dados e a identificação das empresas utilizou-se como fontes de dados complementares as bases de dados Bloomberg e Econondata, especializadas em relatórios de empresas, além dos sites institucionais das empresas identificadas por nome na base de dados do CNPq.

O estudo está estruturado em sete seções principais. A primeira consiste nesta introdução. A segunda seção oferece uma revisão da literatura dividida em duas partes: uma abordagem da indústria farmacêutica com uma indústria baseada em ciência; e uma revisão sobre a evolução recente da indústria farmacêutica brasileira. A terceira seção apresenta as fontes de dados e a descrição da metodologia adotada no estudo. A quarta seção apresenta os resultados da análise. A quinta seção apresenta as considerações finais do artigo, seguida das conclusões e referências bibliográficas utilizadas.

\footnotetext{
${ }^{4}$ Disponível em: < http://www.planalto.gov.br/ccivil_03/leis/L9787.htm>. Acesso em: 25 de julho de 2016.
} 


\section{Revisão da literatura}

\subsection{Uma indústria baseada em ciência.}

A revolução biotecnológica representou para a indústria farmacêutica uma alteração radical na base de conhecimentos e a ruptura do paradigma tecnológico no qual se apoiava a busca por novos compostos terapêuticos. Desde a década de 1980, o setor farmacêutico sofreu profundas transformações que impactaram as condições de entrada na indústria, o tamanho, o comportamento estratégico e as trajetórias de crescimento das firmas. Por um lado, o aumento da intensidade e dos custos P\&D e marketing induziram processo de fusões e aquisições de firmas dedicadas à biotecnologia por parte das grandes corporações da indústria farmacêutica, aumentando a concentração e a globalização da indústria. Por outro lado, observouse a emergência de um novo padrão de colaboração nas cadeias de PD\&I envolvendo articulações entre os agentes com compõem o sistema setorial de inovação farmacêutica: empresas farmacêuticas estabelecidas, empresas dedicadas à biotecnologia para saúde humana, universidades, centros de pesquisa tecnológica e instituições governamentais (FONSECA, 2009; MCKELVEY. ORSENIGO, 2001; REIS et al, 2010).

A indústria farmacêutica e biofarmacêutica é definida na literatura como um setor de atividade baseado em ciência, no qual o processo de inovação é impulsionado por avanços conjuntos em pesquisa básica e aplicada na área de biociências e por progressos em métodos de pesquisa (DOSI. MAZZUCATO, 2006). As empresas baseadas em ciência, por sua vez, são aquelas encontradas em setores cujas fontes da tecnologia são as atividades de pesquisa e desenvolvimento conduzidas em laboratórios corporativos e baseadas no rápido desenvolvimento da ciência acadêmica (PAVITT, 1984). Fonseca (2009) argumenta que o desenvolvimento da base de conhecimentos científico-tecnológicos é pré-condição para o desenvolvimento da indústria de biotecnologia. Em setores baseados em ciência, as inovações são mais intensivas na fase de pesquisa - em relação às fases de desenvolvimento e produção - e o acesso ao conhecimento externo produzido por universidades e institutos públicos de pesquisa é crítico para a inovação. As oportunidades tecnológicas são altas e persistentes e o desenho de novos produtos e processos baseia-se na exploração comercial de um conjunto interdisciplinar de conhecimentos (CORIAT et al, 2003).

Eliasson \& Eliasson (1996) apontam para a necessidade do estabelecimento de canais de comunicação entre invenções geradas no âmbito acadêmico e potenciais aplicações comerciais, através das interações entre as especialidades de cientistas acadêmicos e a experiência comercial dos cientistas alocados em laboratórios corporativos de P\&D para viabilizar o surgimento de inovações disruptivas. Os autores abordam a indústria relacionada à utilização da biotecnologia como uma construção de blocos heterogêneos de conhecimentos que coevoluem no tempo. A origem dessa abordagem reside na interdisciplinaridade e na heterogeneidade da base de conhecimentos científicos e tecnológicos na qual se apoia a biotecnologia moderna. As novas aplicações comerciais das invenções biotecnológicas surgem de combinações de peças de conhecimento científico características de diversas áreas de conhecimento, como, por exemplo: fisiologia, biologia geral, bioquímica, biofísica e biologia molecular.

\footnotetext{
Biotechnology originated in academia and therefore corresponds to the ideal picture of a sciencebased industry pushed in academic literature (...) New discoveries of products are almost always the result of a combination of different clusters of scientific knowhow; for instance in chemistry, in molecular biology, cell biology, physiology and laboratory technology. As a consequence, innovations and industrial applications predominantly occur in competence blocs where a sufficiently diverse environment exists and where new and often not foreseen synergy effects can be captured (ELIASSON. ELIASSON, 1996:7-8)
}

A literatura acadêmica destaca a importância das universidades e centros de pesquisa para a inovação no setor produtivo de acordo com três funções: 1) Ensino e formação de recursos humanos; 2) Pesquisa e expansão da base de conhecimentos; e 3) Empreendedorismo acadêmico (Sampat; Mowery, 2005. Etzkowitz; Leydesdorff, 2000. Paranhos, 2012).

Dentro do arcabouço de Sistemas Nacionais de Inovação, as funções clássicas de ensino e pesquisa são destacadas. A função de ensino está diretamente relacionada à formação de recursos humanos e ao treinamento de pessoal em métodos de pesquisa. O capital humano é considerado um fator essencial para o sucesso do esforço de inovação que se dá dentro das empresas privadas. A contratação de bacharéis, 
graduados e pós-graduados de universidades é considerada uma forma indireta de transferência de conhecimentos da academia para o setor produtivo (Sampat; Mowery, 2005).

Gibbons e Johnston (1974), ao analisarem possíveis contribuições da ciência para a tecnologia, ressaltaram a importância da figura do solucionador de problemas descrita sob a forma de um professional qualificado em métodos de pesquisa capaz de identificar, viabilizar e estimular o acesso às fontes internas e, principalmente, externas de conhecimento para a solução de problemas específicos surgidos ao longo dos processos de produção e inovação.

A segunda função clássica das universidades está relacionada a reposição da base de conhecimentos. A contribuição direta da pesquisa acadêmica para a reposição da base de conhecimento e a expansão de oportunidades tecnológicas é mais propensa a ocorrer em indústrias baseadas em ciência (Klevorick et al.1995). Em setores intensivos em ciência e tecnologia, interações dinâmicas mutuamente benéficas entre empresas e universidade podem ser estabelecidas para gerar, desenvolver e difundir novas tecnologias e inovações em geral (Sampat; Mowery, 2005).

A terceira função da universidade foi proposta dentro do arcabouço teórico do modelo da tripla hélice que enfatiza a atuação empreendedora das universidades (Etzkowitz; Leydesdorff, 2000). No modelo, o sistema de inovação assume o formato de uma estrutura interconectada composta por três esferas dinâmicas e interativas: a esfera acadêmica, o governo e a esfera produtiva. As universidades emergem como uma fonte principal de inovações disruptivas, frente a um caráter mais incremental dos avanços alcançados no âmbito das firmas individuais. Neste contexto, as universidades articulam-se com o setor produtivo: 1) patenteado e licenciado novas peças de conhecimento para o setor produtivo; 2) oferecendo serviços de consultorias e estabelecendo parcerias com empresas; 3) promovendo o surgimento de novos empreendimentos de base tecnológica no formato de start-ups tecnológicas e spin-offs acadêmicos - novas empresas criadas para explorar comercialmente peças de conhecimento geradas na academia (SHANE, 2004) - (Etzkowitz; Leydesdorff, 2000. Sampat; Mowery, 2005).

\subsection{A estrutura da indústria farmacêutica no Brasil}

A estrutura brasileira de produção de fármacos e medicamentos precisa ser analisada conforme um panorama amplo da questão da saúde no Brasil para que algumas características particulares sejam contempladas. O Brasil é marcado pela existência do Sistema Único de Saúde (SUS) de caráter público e universalista pautado pela ideia de que o acesso a saúde é um direito social. A provisão de saúde constitui um elemento estruturante da Estado de Bem-Estar Brasileiro (GADELHA et al 2015).

Por um lado, questões demográficas de crescimento e envelhecimento da população associadas a mudanças nos padrões epidemiológicos, representaram pressões crescentes sobre os gastos públicos em aquisições de medicamentos e o agravamento do padrão de dependência externa crescente da importação de medicamentos de alto valor agregado produzidos por rotas biotecnológicas. Por outro lado, a capacidade do Estado de implementar políticas para a promoção local de medicamentos, tais como a utilização do poder de compra do governo e a promoção de parcerias produtivas entre agentes públicos e privados, atuam como facilitadores para o crescimento e a consolidação da indústria nacional de medicamento (VARGAS et al 2013). Neste contexto, o avanço da produção nacional de fármacos e biofármacos surge com uma questão estratégica nacional, em função da possibilidade de crescimento econômico representada pela inserção produtiva nacional em segmentos de alto valor e do potencial alívio que a nacionalização da produção representa para o balanço comercial do complexo econômico e industrial da saúde no Brasil (ABDI, 2013).

A estrutura produtiva da indústria farmacêutica brasileira configura-se em torno da presença de cinco elementos fundamentais: as grandes empresas farmacêuticas multinacionais instaladas no país, as empresas farmacêuticas nacionais, as firmas dedicadas à biotecnologia para saúde humana, os laboratórios públicos de pesquisa e produção de medicamentos e as universidades e centros de pesquisa acadêmica.

As grandes empresas farmacêuticas multinacionais, que dominam o mercado mundial, têm historicamente uma grande participação no mercado farmacêutico nacional. A partir da década de 1950, muitas empresas começaram a buscar acesso ao mercado brasileiro de medicamentos. Em função da existência à época da política brasileira de substituição de importações que visava proteger a indústria 
nascente brasileira contra a concorrência representada pelos fluxos de importação, muitas grandes empresas farmacêuticas optaram por abrir filiais em solo brasileiro. Apesar de trazerem ao país as etapas de produção e distribuição, as empresas multinacionais mantiveram seus esforços inovadores concentrados em suas matrizes no exterior. As atividades PD\&I das empresas multinacionais no país restringiram-se à adaptação da produção à utilização de insumos locais e à adequação às exigências fitossanitárias brasileiras. Com isso, as grandes assimetrias tecnológicas e barreiras à entrada no setor farmacêutico tenderam a se perpetuar. Observou-se ao longo das décadas de 1970 e 1980, o predomínio das empresas multinacionais no mercado farmacêutico nacional, período em que essas empresas dominavam uma parcela entre $75 \%$ e $85 \%$ do mercado brasileiro de medicamentos (PARANHOS, 2012).

A introdução no país da lei dos Genéricos, lei n $9789 / 1999$, representou um ponto de inflexão para a indústria farmacêutica nacional ao regular o registro de cópias de medicamento não protegidos por patentes mediante aprovação em testes de bioequivalência e biodisponibilidade em laboratórios analíticos brasileiros certificados pela Agência Nacional de Vigilância Sanitária (ANVISA). A introdução do segmento de genéricos resultou em um grande aumento da participação nacional no mercado farmacêutico local, além de impulsionar processos de aprendizagem e a construção de competências das empresas farmacêuticas nacionais (PARANHOS, 2012). Estima-se que a participação das farmacêuticas nacionais tenha subido de 32\% do mercado brasileiro de medicamentos em 2003 para mais de $50 \%$ do mercado no ano 2010 (GADELHA et al 2012).

Em 1999, a Lei 9.787, conhecida como a Lei de Genéricos, estabeleceu a necessidade de testes de bioequivalência e biodisponibilidade a serem realizados na Rede Brasileira de Laboratórios Analíticos em Saúde para registro de medicamentos cópia de medicamentos sem patente, instituindo no Brasil o segmento de medicamentos genéricos. Esta mudança institucional serviu como alternativa e estímulo ao desenvolvimento das empresas nacionais. Desde então, a participação destas e o crescimento do mercado é constante. Em abril de 2014, o mercado de genéricos alcançou $28 \%$ em volume do mercado farmacêutico nacional, sendo que as empresas brasileiras são tradicionalmente responsáveis por cerca de $90 \%$ dos medicamentos genéricos consumidos no país (Prógenéricos, 2015). Com o fortalecimento deste segmento, as empresas nacionais ganharam porte e recursos para novos investimentos, que parecem cada vez mais estar sendo direcionados a atividades inovativas, como visto nos dados analisados acima (PARANHOS. HASENCLEVER., 2015 p. 9)

Apesar dos avanços alcançados pelas empresas farmacêuticas nacionais desde a implementação da lei dos genérico em 1999, a indústria farmacêutica nacional ainda apresenta gargalos estruturais na condução de processos internos de PD\&I e na inserção no segmento de drogas terapêuticas produzidas por rotas biotecnológicas (VARGAS et al., 2013). Conforme apontado por Paranhos (2012), a estratégia de crescimento através da produção de genéricos limita a capacidade de inovação das empresas que tendem a investir pouco em esforços de PD\&I. Em geral, o conhecimento necessário para a fabricação de medicamentos genéricos costuma estar bem codificada nos documentos de patentes.

Paranhos e Hasenclever (2014), entretanto, identificaram uma mudança no padrão de condução de esforços inovativos pelas empresas brasileiras a partir do ano 2008, marcado pela ampliação das capacidades internas e da criação de conhecimento das firmas. Segundo as autoras, o número de empresas que realizam atividades internas de $P \& D$ e o valor gasto nestas atividades apresentaram aumentos continuados entre os anos 2008 e 2011.

Gomes (2014) destaca a inserção das empresas farmacêuticas nacionais especializadas na produção de genéricos no seguimento de medicamentos biológicos. Segundo o autor, o Brasil teria optado por uma estratégia de inserção nos segmentos de biofármacos através de um modelo de grandes empresas campeãs setoriais e de formação de joint-ventures de empresas farmacêuticas nacionais. As empresas contam com o apoio do governo brasileiro através de uma política de Parcerias para o Desenvolvimento Produtivo (PDD) aliada a políticas de compra garantida e de financiamento pelo Banco Nacional de Desenvolvimento Econômico e Social (BNDES) e pela Financiadora de Estudos e Projetos (FINEP).

A política de Parcerias para o Desenvolvimento Produtivo (PDP) coordenada pelo ministério da saúde foi desenhada para promover a transferência de tecnológica entre laboratórios públicos e empresas farmacêuticas privadas com o objetivo de internalizar a produção de fármacos, biofármacos e equipamentos médicos considerados estratégicos pelos formuladores de política. 
A política das PDPs vem sendo combinada com uma política de compras governamentais, regulamentada pela lei $\mathrm{n}^{\mathrm{0}} 12.349$ e pela medida provisória $\mathrm{n}^{\circ}$ 495, que prevê a adoção de uma margem de preferência de até $25 \%$ na compra de para medicamentos estratégicos produzidos no Brasil (VARGAS et. al.,2012; VARGAS.BRITTO, 2015).

Gomes (2014) aponta dois argumentos que sustentam a opção pelas grandes empresas. Em primeiro lugar, o país teria supostamente optado pela criação de empresas âncora, capazes de gerar incentivos para o surgimento de pequenas empresas de biotecnologia dedicadas à condução de P\&D. Em segundo lugar, a própria lógica das PDPs baseada na ação do governo - atuando como monopsonista - no estimulo à produção através de licitações de compras governamentais estaria necessariamente associada à existência de economias de escala em produção e distribuição.

Outro aspecto importante da inserção das empresas farmacêuticas nacionais no segmento de biofármacos é a estratégia de produção de medicamentos biossimilares ou bioequivalentes. Os medicamentos biossimilares podem ser definidos como "quase cópias" dos biofármacos originais com patentes expiradas. Em função das especificidades tecnológicas envolvidas na produção de biológicos, não é possível a replicação perfeita do medicamento com base da informação codificada nos documentos de patente. Logo a realização de cópias desses medicamentos exige que as empresas possuam competências e capacitações científicas e tecnológicas na produção de drogas por rotas biotecnológicas. Entretanto, assim como no caso dos medicamentos genéricos, a estratégia de produção biossimilares funciona como um limitante da capacidade inovação das empresas por ser pautada por um caráter de melhorias incrementais realizadas ao longo de processo de replicação (GOMES, 2014).

Conforme indicado por Gomes (2014), existe uma polêmica envolvida na dicotomia criada pelo modelo brasileiro de inserção em biofármacos através de grandes empresas e o modelo de pequenas empresas, spin-offs ou start-ups, descrito na literatura e nas estatísticas mundiais sobre biotecnologia para saúde humana.

Alguns estudos - Biominas e PWC (2011), e CEBRAP e BRBIOTEC (2011) e Bianchi (2012) indicam que o Brasil possui inúmeras empresas de biotecnologia constituídas segundo o modelo de micro e pequenas empresas de base tecnológica referenciado na literatura mundial.

Não há um consenso sobre o número exato de empresas de biotecnologia em atividade no país, em função das dificuldades envolvidas na identificação de empresas atuando em uma atividade altamente interdisciplinar. Entretanto, estima-se que o Brasil possua entre 31 e 94 empresas de biotecnologia voltadas à saúde humana. Em sua maioria, essas empresas são: pequenas ou médias; jovens; controladas majoritariamente por capital nacional; concentradas nas regiões sul e sudeste; apoiadas por algum tipo de financiamento público e altamente relacionadas à universidades e centros de pesquisa. Também há um consenso sobre o envolvimento dessas empresas em atividades de alto risco e essencialmente inovadoras, com um maior grau de inovações disruptivas.

Os laboratórios públicos de pesquisa e produção - como, por exemplo, o Instituto Biomanguinhos e o Instituto Butantan - constituem o quarto elo da estrutura farmacêutica nacional (ABDI, 2013). O Brasil conta com 16 laboratórios públicos em funcionamento que são responsáveis por cerca de $3 \%$ em valor da produção nacional de fármacos e medicamentos e 10\% em volume de produção. Os laboratórios oficiais são, ainda, responsáveis por atender cerca de $80 \%$ da demanda doméstica por vacinas (GADELHA et al, 2012; VARGAS et. Al, 2013).

As universidades e o centros de pesquisa constituem o quinto e último elo integrante da estrutura farmacêutica brasileira. Muito embora não se destinem a produção direta de produtos e serviços farmacêuticos, a inclusão das instituições acadêmicas justifica-se pelas importantes funções de pesquisa e de capacitação desempenhada por esses centros. Outro aspecto elementar é a atuação dessas instituições no formato de elos de ligação entre informações e agentes organizacionais do sistema setorial de inovação. Albuquerque e Cassiolato (2002) argumentam que além da formação de profissionais, as universidades agem com um centro de convergência de fluxos de informação provenientes não apenas de suas próprias pesquisas, mas também de interações com empresas, agências regulatórias, hospitais, clínicas, postos médicos entre outros atores capazes de transmitir novas demandas à indústria farmacêutica.

O Brasil possui uma ampla gama de instituições acadêmicas com grupos de pesquisa consolidados em ciências biológicas e da saúde. Em termos de capacitações, observou-se no país um aumento 
significativo no número de pesquisadores em cursos de pós-graduação em biociências ao longo do período que compreende os anos de 2000 a 2010, destacando-se o papel desempenhado pelos programas da Coordenação de Aperfeiçoamento de Pessoal de Nível Superior (CAPES) e do Conselho Nacional de Desenvolvimento Científico e Tecnológico (CNPq) para a formação de pessoal em métodos e técnicas avançadas (GUIMARÃES, 2011; BRITTO et al., 2012). Britto et al. (2012) apontam que o número de grupos de pesquisa concentrados nas grandes áreas de ciências da saúde e ciências biológicas cresceu de 3.790 grupos no ano 2000 para 8.179 grupos no ano 2014, refletindo um crescimento de $115 \%$ no período analisado. Ao passo que o número de grupos das áreas de ciências biológicas e da saúde que estabeleceram relacionamentos com o setor produtivo cresceu 221\% entre os anos 2000 e 2010, passando de 274 grupos interativos em 2000 para 879 em 2010. Com o objetivo de avaliar a capacidade nacional de geração de conhecimento científico na área de biotecnologia, Bianchi (2012) identificou 686 grupos envolvidos em atividades de biotecnologia, dos quais 230 estariam voltados às atividades de biotecnologia moderna.

\section{Metodologia e base de dados}

Análise exploratória sobre o perfil dos elementos que compões a indústria farmacêutica baseou-se na utilização de dados extraídos do Censo 2010 do Diretório Nacional dos Grupos de Pesquisa do Conselho Nacional de Desenvolvimento Científico e Tecnológico (CNPq). Os dados foram filtrados por ramo de atividade. Selecionou-se os ramos de atividade: fabricação de produtos farmoquímicos e farmacêuticos e pesquisa e desenvolvimento de científico ( $P \& D$ científico). A justificativa para a inclusão do ramo de atividade descrito como $P \& D$ científico consiste no fato de que as empresas dedicadas à biotecnologia e alguns laboratórios oficiais de pesquisa e produção de fármacos e medicamentos estão catalogados sob este ramo de atividade, ao passo que as empresas farmacêuticas são catalogam sob o ramo de fabricação de produtos farmoquímicos e farmacêuticos.

O censo 2010 é a fonte de dados mais recente disponível no plano tabular do diretório do CNPq para a série de dados selecionados. Os dados disponíveis permitem a avaliação das empresas conforme tipos de interação estabelecidos com os grupos de pesquisa, número de funcionários, natureza jurídica e dispersão geográfica por regiões e unidades da federação. Não há ocorrência de problemas de dupla contagem nos dados desagregados sobre empresas e grupos de pesquisa, mas pode haver dupla contagem nos totais obtidos por soma. A classificação por ramo de atividade é realizada pelo CNPq com base nas informações da Classificação Nacional de Atividade Econômica (CNAE). Os dados sobre natureza jurídica e faixa de pessoal ocupado são obtidos do Instituto Brasileiro de Geografia e Estatística (IBGE) pelo CNPq e divulgados no Diretório dos Grupos de Pesquisa.

Para a atualização dos dados e a identificação das empresas utilizou-se como fontes de dados complementares as bases de dados Bloomberg e Econondata, especializadas em relatórios de empresas, além dos sites institucionais das empresas identificadas por nome na base de dados do CNPq.

\section{Resultados}

O diretório dos grupos de pesquisa do CNPq permitiu a identificação de 243 empresas. Destas, 103 empresas são classificadas sob o ramo de atividade definido como fabricação de produtos farmoquímicos e farmacêuticos e 140 empresas sob o ramo de P\&D científico. Essas empresas mantiveram relacionamentos ativos com 492 grupos de pesquisa cadastrados no CNPq, conforme mostrado na Tabela 1. Observa-se que $42 \%$ da amostra de empresas consistia em empresas farmacêuticas ou farmoquímicas, e essas empresas interagiram com $28 \%$ dos grupos de pesquisa identificados. Ao passo que as empresas de P\&D científico corresponderam 58\% da amostra de empresas e relacionaram-se com $72 \%$ dos grupos de pesquisa da amostra.

Um índice para medir a importância das interações universidade-empresa para cada ramo de atividade foi criado dividindo-se o número de grupos de pesquisa pelo número de empresas. Estima-se que cada empresa farmacêutica ou farmoquímica relacionou-se com 1,3 grupos de pesquisa, ao passo que a intensidade dessas interações para o ramo de P\&D científico foi superior, refletida no índice de 2,5 grupos

\footnotetext{
${ }^{5}$ Com base em uma triagem a partir dos dados da base corrente do Diretório de Grupos de pesquisa do CNPq (BIANCHI, 2012).
} 
de pesquisa por empresa. Portanto, há indícios de maior intensidade dos relacionamento universidadeempresa para o ramo de $P \& D$ científico, em comparação à ao ramo de atividade que engloba empresas farmacêuticas e farmoquímicas.

Quando comparamos a amostra selecionada com o universo disponível na base do CNPQ, descobrimos de $5 \%$ das empresas catalogadas na base de dados interage com $8 \%$ dos grupos de pesquisa interativos. Enquanto o universo do CNPq indica uma média de 1,3 grupos de pesquisa interativos por empresa, a amostra selecionada reflete uma média de 2 grupos de pesquisa por empresa. A maior interação dos ramos de atividades selecionados em comparação com a base do CNPq é um resultado esperado e condizente com a literatura, em função da importância das interações com universidades para indústrias baseadas em ciência ${ }^{6}$.

\begin{tabular}{|c|c|c|c|c|}
\hline Ramo de atividade & Empresas & $\%$ & Grupos & $\%$ \\
\hline Fabricação de produtos farmoquímicos e farmacêuticos & 103 & $42 \%$ & 137 & $28 \%$ \\
\hline Pesquisa e desenvolvimento científico & 140 & $58 \%$ & 355 & $72 \%$ \\
\hline Total selecionados & 243 & & 492 & \\
\hline Total CNPQ & 4995 & & 6259 & \\
\hline Selecionados/CNPq & $5 \%$ & & $8 \%$ & \\
\hline
\end{tabular}

Fonte: Elaboração própria com base nos dados do Diretório dos Grupos de Pesquisa do CNPq

Os dados permitem a análise das interações universidade-empresa por tipo de relacionamento. Foram identificados 943 relacionamentos entre as empresas e os grupos de pesquisa pertencentes aos ramos de atividade selecionados. Esses relacionamentos correspondem a 7\% dos 13.113 tipos de relacionamentos divulgados no censo 2010 do diretório do CNPq, conforme mostrado na Tabela 2. É importante ressaltar que a base de dados do CNPq permite a contabilização de até três tipos de interação por grupo de pesquisa, podendo haver erros de dupla contagem nos dados. Os tipos de interação mais frequentes nos ramos de atividade selecionados foram: os esforços conjuntos na condução de pesquisa básica (19\%) e aplicada (36\%) e as atividade de transferência de tecnologia (16\%).

A comparação entre os dois ramos de atividade selecionados indica que $32 \%$ dos relacionamentos envolveram empresas farmoquímicas e farmacêuticas, enquanto os demais $68 \%$ das interações envolveram empresas dedicadas à $P \& D$ científico. A condução conjunta de pesquisa básica é proporcionalmente mais relevante para o ramo de $P \& D$ científico, ao passo que a condução de pesquisa aplicada e a transferência de tecnóloga são ligeiramente mais significativas para o ramo de fabricação de produtos farmoquímicos e farmacêuticos. Um destaque é a importância do fornecimento de insumos, juntamente com a transferência de tecnologia, para o ramo de farmoquímica e farmacêutica.

Tabela 2- Tipo de relacionamento segundo ramo de atividade da empresa, Censo 2010.

\begin{tabular}{|c|c|c|c|c|c|c|c|c|c|}
\hline Tipo de relacionamento & $\begin{array}{l}\text { Fabricação de } \\
\text { farmoquímicos } \\
\text { e farmacêuticos }\end{array}$ & $\%$ & $\begin{array}{r}\mathrm{P} \& \mathrm{D} \\
\text { científico }\end{array}$ & $\%$ & $\begin{array}{r}\text { Total ramos } \\
\text { de atividade } \\
\text { selecionados }\end{array}$ & $\%$ & $\begin{array}{r}\text { Total } \\
\text { CNPq }\end{array}$ & $\%$ & $\begin{array}{r}\text { Ramos } \\
\text { selecionados/ } \\
\text { CNPQ } \\
\end{array}$ \\
\hline Pesquisa básica & 44 & $14 \%$ & 139 & $22 \%$ & 183 & $19 \%$ & 1995 & $15 \%$ & $9 \%$ \\
\hline Pesquisa aplicada & 120 & $39 \%$ & 217 & $34 \%$ & 337 & $36 \%$ & 3813 & $29 \%$ & $9 \%$ \\
\hline Engenharia não rotineira & 4 & $1 \%$ & 25 & $4 \%$ & 29 & $3 \%$ & 593 & $5 \%$ & $5 \%$ \\
\hline Desenvolvimento de software & 1 & $0 \%$ & 10 & $2 \%$ & 11 & $1 \%$ & 426 & $3 \%$ & $3 \%$ \\
\hline Transferência de tecnologia & 62 & $20 \%$ & 85 & $13 \%$ & 147 & $16 \%$ & 2136 & $16 \%$ & $7 \%$ \\
\hline Consultoria técnica & 20 & $7 \%$ & 38 & $6 \%$ & 58 & $6 \%$ & 978 & $7 \%$ & $6 \%$ \\
\hline Fornecimento de insumos & 32 & $10 \%$ & 39 & $6 \%$ & 71 & $8 \%$ & 1123 & $9 \%$ & $6 \%$ \\
\hline Treinamento de pessoal & 5 & $2 \%$ & 47 & $7 \%$ & 52 & $6 \%$ & 1099 & $8 \%$ & $5 \%$ \\
\hline Outros tipos & 17 & $6 \%$ & 38 & $6 \%$ & 55 & $6 \%$ & 950 & $7 \%$ & $6 \%$ \\
\hline Total dos relacionamentos & 305 & & 638 & & 943 & & 13113 & & $7 \%$ \\
\hline
\end{tabular}

Fonte: Elaboração própria com base nos dados do Diretório dos Grupos de Pesquisa do CNPq

Utilizando-se o critério de número de empregados como um indicativo para o tamanho da empresa, conforme proposto pelo Serviço Brasileiro de Apoio às Micro e Pequenas Empresas (SEBRAE, 2011), consideram-se: microempresas aquelas que empregam até 19 funcionários; pequenas aquelas com 20 a 99

${ }^{6}$ Ver Klevorick et al (1995). 
funcionários; médias as empresas com 100 a 499 funcionários; e grandes as empresas com mais de 500 funcionários.

Estima-se que 86\% das empresas catalogadas sob os ramos de atividade selecionados sejam micro, pequenas e médias empresas. Apenas 14\% das empresas selecionadas possuíam mais de 500 funcionários. O ramo de $\mathrm{P} \& \mathrm{D}$ científico concentrou a maior parcela de micro e pequenas empresas, cerca de $82 \%$ de suas 140 empresas. Ao passo que o ramo de fabricação de produtos farmoquímicos e farmacêuticos concentrou 76\% das 34 grandes empresas e 59\% das 41 médias empresas contidas na amostra (Tabela 3).

\begin{tabular}{|c|c|c|c|c|c|c|c|c|}
\hline \multirow[b]{2}{*}{ Pessoal ocupado } & \multicolumn{2}{|c|}{$\begin{array}{c}\text { Fabricação de produtos } \\
\text { farmoquímicos e farmacêuticos }\end{array}$} & \multicolumn{2}{|c|}{$\begin{array}{l}\text { Pesquisa e desenvolvimento } \\
\text { científico }\end{array}$} & \multicolumn{2}{|c|}{$\begin{array}{c}\text { Total ramos de } \\
\text { atividade selecionados }\end{array}$} & \multirow[b]{2}{*}{$(\mathrm{A} / \mathrm{T}) \%$} & \multirow[b]{2}{*}{$(\mathrm{B} / \mathrm{T}) \%$} \\
\hline & Empresas (A) & $\%$ & Empresas (B) & $\%$ & Empresas (T) & $\%$ & & \\
\hline Até 19 & 23 & $22 \%$ & 90 & $64 \%$ & 113 & $47 \%$ & $9 \%$ & $37 \%$ \\
\hline 20 a 49 & 14 & $14 \%$ & 14 & $10 \%$ & 28 & $12 \%$ & $6 \%$ & $6 \%$ \\
\hline 50 a 99 & 16 & $16 \%$ & 11 & $8 \%$ & 27 & $11 \%$ & $7 \%$ & $5 \%$ \\
\hline 100 a 499 & 24 & $23 \%$ & 17 & $12 \%$ & 41 & $17 \%$ & $10 \%$ & $7 \%$ \\
\hline 500 e mais & 26 & $25 \%$ & 8 & $6 \%$ & 34 & $14 \%$ & $11 \%$ & $3 \%$ \\
\hline TOTAIS & 103 & & 140 & & 243 & & $42 \%$ & $58 \%$ \\
\hline
\end{tabular}

Fonte: Elaboração própria com base nos dados do Diretório dos Grupos de Pesquisa do CNPq

Uma outra característica observável sobre o perfil das empresas selecionadas é a elevada concentração regional dessas empresas. Cerca de 56\% das empresas localizavam-se na região sudeste, $18 \%$ na região sul e $14 \%$ na região nordeste. As regiões norte e centro-oeste juntas correspondiam a apenas $11 \%$ das empresas catalogadas sob os ramos de atividade selecionados. É preciso mencionar que existe um problema de dupla contagem envolvido nas estatísticas sobre concentração regional, em função da menção de uma mesma empresa com mais de umas filiais distribuídas pelo país. Entretanto, esse erro gerou uma distorção de 7 empresas que pode ser considerado pequeno frente às 243 identificadas sem dupla contagem.

Com base nos percentuais mostrados na Tabela 4, calculou-se um índice de concentração conhecido como Herfindahl-Hirschman Index (HHI), somando-se os quadrados dos percentuais de empresas por região para cada ramo de atividade. Quanto maior e mais próximo de 1 for o valor do HHI, maior é a concentração do ramo de atividade. Os índices obtidos foram 0,43 para as empresas farmoquímicas e farmacêuticas e 0,35 para empresas de $P \& D$ científico, indicando uma maior concentração das primeiras em relação às segundas.

\begin{tabular}{|c|c|c|c|c|}
\hline \multirow{2}{*}{$\frac{\text { Região geográfica }}{\text { Centro-Oeste }}$} & \multicolumn{2}{|c|}{$\begin{array}{l}\text { Fabricação de produtos } \\
\text { farmoquímicos e farmacêuticos }\end{array}$} & \multicolumn{2}{|c|}{ Pesquisa e desenvolvimento científico } \\
\hline & 8 & $8 \%$ & 9 & $6 \%$ \\
\hline Nordeste & 10 & $10 \%$ & 26 & $18 \%$ \\
\hline Norte & 1 & $1 \%$ & 9 & $6 \%$ \\
\hline Sudeste & 63 & $61 \%$ & 78 & $53 \%$ \\
\hline Sul & 21 & $20 \%$ & 25 & $17 \%$ \\
\hline $\mathrm{HHI}$ & & 0,43 & 0,35 & 0,35 \\
\hline
\end{tabular}

Fonte: Elaboração própria com base nos dados do Diretório dos Grupos de Pesquisa do CNPq

Os dados disponíveis no Diretório do CNPq permitem, ainda, avaliar a concentração espacial das empresas por unidades da federação, conforme mostrado na tabela 5. Observa-se que o ramo de P\&D científico possui empresas espalhadas por um número maior de estados (23 estados) em comparação ao ramo de produção farmacêutica e farmoquímica (14 estados).

Um grande destaque é a importância do Estado de São Paulo que concentra $42 \%$ das empresas farmoquímicas e farmacêuticas e 34\% das empresas de P\&D científico. Os estados mais significativos por ramo de atividade foram: Goiás, Pernambuco, Minas Gerais, Rio de Janeiro, São Paulo, Paraná e Rio Grande do Sul para o ramo de fabricação de produtos farmoquímicos e farmacêuticos; e Bahia, Pernambuco, Minas Gerais, Rio de Janeiro, São Paulo, Paraná e Rio Grande do Sul para o ramo de P\&D científico.

O índice HHI foi novamente calculado para avaliar a concentração das empresas por unidade federativa para cada um dos ramos de atividade. Os índices confirmam a maior dispersão geográfica do 
ramo de $\mathrm{P} \& \mathrm{D}$ científico $(\mathrm{HHI}=0,15)$ em relação à produção de farmacêuticos e farmoquímicos $(\mathrm{HHI}=$ 0,22 ) em termos de distribuição das empresas por unidade federativa. Uma explicação possível para a maior dispersão geográfica do ramo do ramo de $P \& D$ científico é a existência de empresas sem fins lucrativos e organizações governamentais de amparo à ciência e tecnologia classificadas sob este ramo de atividade, como por exemplo filiais da Embrapa espalhadas por todo país.

\begin{tabular}{|c|c|c|c|c|c|c|c|}
\hline \multicolumn{4}{|c|}{$\begin{array}{l}\text { Fabricação de produtos farmoquímicos e } \\
\text { farmacêuticos }\end{array}$} & \multicolumn{4}{|c|}{ Pesquisa e desenvolvimento científico } \\
\hline & $\begin{array}{l}\text { No de } \\
\text { empresas }\end{array}$ & \% Região & $\%$ Brasil & & $\begin{array}{c}\mathrm{N}^{\circ} \text { de } \\
\text { empresas }\end{array}$ & \% Região & $\%$ Brasil \\
\hline \multicolumn{8}{|c|}{ Centro-Oeste } \\
\hline Distrito Federal & 1 & $13 \%$ & $1 \%$ & Distrito Federal & 3 & $27 \%$ & $2 \%$ \\
\hline Goiás & 7 & $88 \%$ & $7 \%$ & Goiás & 5 & $45 \%$ & $3 \%$ \\
\hline- & - & & & Mato Grosso do Sul & 3 & $27 \%$ & $2 \%$ \\
\hline \multicolumn{8}{|c|}{ Nordeste } \\
\hline- & - & 10 & & Alagoas & 2 & $6 \%$ & $1 \%$ \\
\hline Bahia & 1 & $10 \%$ & $1 \%$ & Bahia & 9 & $28 \%$ & $5 \%$ \\
\hline Ceará & 1 & $10 \%$ & $1 \%$ & Ceará & 5 & $16 \%$ & $3 \%$ \\
\hline Paraíba & 2 & $20 \%$ & $2 \%$ & Paraíba & 3 & $9 \%$ & $2 \%$ \\
\hline Pernambuco & 5 & $50 \%$ & $5 \%$ & Pernambuco & 8 & $25 \%$ & $5 \%$ \\
\hline Piauí & 1 & $10 \%$ & $1 \%$ & Piauí & 2 & $6 \%$ & $1 \%$ \\
\hline- & - & & & Rio Grande do Norte & 1 & $3 \%$ & $1 \%$ \\
\hline- & - & & & Sergipe & 2 & $6 \%$ & $1 \%$ \\
\hline \multicolumn{8}{|c|}{ Norte } \\
\hline- & - & & & Acre & 1 & $8 \%$ & $1 \%$ \\
\hline Amazonas & 1 & $100 \%$ & $1 \%$ & Amazonas & 4 & $33 \%$ & $2 \%$ \\
\hline- & - & & & Pará & 2 & $17 \%$ & $1 \%$ \\
\hline- & - & & & Rondônia & 2 & $17 \%$ & $1 \%$ \\
\hline- & - & & & Roraima & 1 & $8 \%$ & $1 \%$ \\
\hline- & - & & & Tocantins & 2 & $17 \%$ & $1 \%$ \\
\hline \multicolumn{8}{|c|}{ Sudeste } \\
\hline Minas Gerais & 10 & $16 \%$ & $10 \%$ & Minas Gerais & 14 & $17 \%$ & $9 \%$ \\
\hline Rio de Janeiro & 10 & $16 \%$ & $10 \%$ & Rio de Janeiro & 12 & $15 \%$ & $7 \%$ \\
\hline São Paulo & 44 & $69 \%$ & $42 \%$ & São Paulo & 56 & $68 \%$ & $34 \%$ \\
\hline \multicolumn{8}{|c|}{ Sul } \\
\hline Paraná & 8 & $38 \%$ & $8 \%$ & Paraná & 12 & $44 \%$ & $7 \%$ \\
\hline Rio Grande do Sul & 8 & $38 \%$ & $8 \%$ & Rio Grande do Sul & 9 & $33 \%$ & $5 \%$ \\
\hline Santa Catarina & 5 & $24 \%$ & $5 \%$ & Santa Catarina & 6 & $22 \%$ & $4 \%$ \\
\hline
\end{tabular}

Fonte: Elaboração própria com base nos dados do Diretório dos Grupos de Pesquisa do CNPq

Ao analisar a natureza jurídica das empresas e instituições envolvidas no relacionamento com universidades, percebemos a existência de entidades sem fins lucrativos e instituições da administração pública no ramo de $\mathrm{P} \& \mathrm{D}$ científico. Diversos institutos de apoio à ciência e tecnologia figuram nas listas de instituições da administração pública e entidades empresariais sem fins lucrativos. A partir dessa informação, é possível reduzir a amostra para as 177 entidades empresariais, conforme mostrado na Tabela 6.

Entre as listas de instituições pertencentes à administração pública, destaca-se a presença da Fundação Oswaldo Cruz. A Fiocruz, como também é conhecida, representa um elemento oficial de elevada relevância dentro do sistema farmacêutico nacional. A estrutura da Fiocruz engloba um importante laboratório oficial, o Instituto de Tecnologia em Imunobiológicos Bio-Manguinhos fundado em 1976. O instituto destaca-se nas atividades de pesquisa e produção de vacinas, medicamentos, reagentes e kits de diagnóstico para atender as demandas da saúde pública no Brasil. Além da relevância nacional, o laboratório Bio-Manguinhos exporta sua produção excedente para cerca de 70 países, colocando em evidência o potencial para a inserção virtuosa do país na produção de biológicos ${ }^{7}$.

Um segundo destaque é a presença da Fundação Bio Rio compreendida na lista de entidades sem fins lucrativos. A Bio Rio é um parque tecnológico, instalado dentro da Universidade Federal do Rio de

\footnotetext{
${ }^{7}$ Conforme informações públicas divulgadas no site institucional da Fundação Oswaldo Cruz e do Instituto Bio-Manguinhos.
} 
Janeiro, dedicado à promoção de pesquisa, desenvolvimento e iniciativas de empreendedorismo em biotecnologia e biociências. Atualmente a Bio Rio abriga 41 empresas (20 na incubadora de empresas e 21 no parque tecnológico), além de possuir parcerias com cerca de 200 instituições nacionais e internacionais, configurando-se em uma importante ponte de integração entre a infraestrutura científica e tecnológica, parceiros do setor produtivo e órgãos oficiais de fomento à tecnologia e inovação em ciências da vida ${ }^{8}$.

\begin{tabular}{|c|c|c|c|c|c|c|}
\hline & \multicolumn{2}{|c|}{$\begin{array}{l}\text { Fabricação de produtos } \\
\text { farmoquímicos e farmacêuticos }\end{array}$} & \multicolumn{2}{|c|}{$\begin{array}{c}\text { Pesquisa e } \\
\text { desenvolvimento } \\
\text { científico }\end{array}$} & \multicolumn{2}{|c|}{$\begin{array}{c}\text { Total ramos de atividade } \\
\text { selecionados }\end{array}$} \\
\hline & Empresas & $\%$ & Empresas & $\%$ & Empresas & $\%$ \\
\hline Administração Pública & - & & 5 & $4 \%$ & 5 & $2 \%$ \\
\hline Entidades Empresariais & 103 & $100 \%$ & 74 & $53 \%$ & 177 & $73 \%$ \\
\hline $\begin{array}{l}\text { Entidades sem Fins } \\
\text { Lucrativos }\end{array}$ & - & & 61 & $44 \%$ & 61 & $25 \%$ \\
\hline TOTAIS & 103 & & 140 & & 243 & \\
\hline
\end{tabular}

Fonte: Elaboração própria com base nos dados do Diretório dos Grupos de Pesquisa do CNPq

Uma análise cuidadosa da lista de 177 entidades empresariais foi conduzida utilizando os dados disponíveis nas bases de dados Econodata e Bloomberg e as informações contidas nos sites institucionais das empresas listadas pelo Censo 2010. Essa análise indicou que 113 entidades empresariais estavam voltadas à saúde humana, o que corresponde a 64\% das 177 entidades empresariais. Cerca de 64 empresas dedicavam-se à saúde animal, pesquisa agropecuária, biocombustíveis, soluções para o meio ambiente e outras atividades de pesquisa e desenvolvimento científico. A seleção não é mutuamente excludente. Por um lado, há registro de empresas com atuação tanto em saúde humana quanto em saúde animal. Por outro lado, três empresas não puderam ser perfeitamente identificadas com base em seus nomes em função de ausência de dados.

Entre as entidades empresariais voltadas à saúde humana constam o nome de cinco laboratórios ou institutos oficiais, a saber: Laboratório Farmacêutico do Estado de Pernambuco, Indústria Química do Estado de Goiás Ltda, Empresa Brasileira de Hemoderivados e Biotecnologia Hemobrás, Instituto Vital Brasil e o Instituto de Tecnologia do Paraná.

É preciso destacar a presença de 36 entidades empresariais dedicadas à produção de medicamentos fitoterápicos e suplementos alimentares. Algumas dessas entidades também atuam no seguimento de medicamentos sob prescrição médica.

O estudo prospectivo permitiu a identificação de 50 empresas farmacêuticas, sendo 29 empresas farmacêuticas nacionais e 19 empresas farmacêuticas multinacionais ${ }^{9}$. O critério utilizado para a classificação foi a localização da matriz controladora da empresa no Brasil ou no exterior. Entre as empresas farmacêuticas nacionais que mantiveram relacionamentos com grupos de pesquisa do CNPq destacam-se a Aché Laboratórios Farmacêuticos, a Eurofarma Laboratórios e o Grupo EMS - empresas destacadas por estarem entre as dez maiores empresas brasileiras do setor farmacêutico segundo Gadelha et al (2012). Entre as empresas multinacionais, destacam-se nomes como Medley, Merck, Novartis e Eli Lilly do Brasil - empresas listadas entre as maiores empresas farmacêuticas do mundo (GADELHA et al 2012).

Identificou-se, ainda, 31 entidades empresariais relacionadas à utilização de biotecnologia, majoritariamente brasileiras e constituídas sob o formado de micro e pequenas empresas de base tecnológica. É preciso ressaltar que diversas empresas farmacêuticas instaladas no país possuem iniciativas em medicamentos biológicos e biossimilares, essas empresas não estão incluídas na lista de 31 empresas em função de dificuldades na identificação de quais empresas farmacêuticas efetivamente estão inseridas em biológicos.

A identificação das atividades em biotecnologia é difícil e controversa por se tratar de uma atividade altamente interdisciplinar. Buscou-se empresas com iniciativas em pesquisa e desenvolvimento de

\footnotetext{
${ }^{8}$ Conforme informações divulgadas no site institucional da Fundação Bio-Rio.

${ }^{9}$ Não foi possível a classificação de duas empresas entre as categorias nacional ou multinacional em função do envolvimento das empresas em operações de fusões e aquisições acionárias.
} 
hormônios, antirretrovirais, biomateriais, reagentes, kits de diagnóstiscos, hemoderivados e pesquisas com células tronco, síntese de proteínas, desenvolvimento de anticorpos monoclonais, técnicas de DNA recombinante, medicamentos para tratamento oncológico e o desenvolvimento de fármacos e medicamentos com base no descobrimento de novas moléculas.

Na lista 31 entidades empresariais encontram-se 3 laboratórios oficiais de pesquisa e produção, 1 empresa pública (a Hemobrás), 1 empresa multinacional (Diamed Latino América) e 26 empresas brasileiras de base tecnológica. De acordo com os dados do Diretório do CNPq, estima-se que 81\% dessas empresas possuem até 19 funcionários e 68\% estão localizadas na região sudeste. Estima-se, ainda, que pelo menos 10 desses empreendimentos estejam envolvidos com pesquisa e desenvolvimento de anticorpos monoclonais, síntese de proteínas, tratamentos oncológicos e de DNA recombinante. Entre as empresas identificadas, destaca-se o caso da Recepta Biopharma.

A Recepta Biopharma é uma empresa brasileira dedicada à pesquisa e ao desenvolvimento de terapias oncológicas direcionadas, baseadas em técnicas de anticorpos monoclonais e ligações peptídicas que atuam diretamente sobre células cancerígenas. A empresa foi criada em 2006 pelo doutor José Fernando Perez em parceria com o Instituto Ludwig de Pesquisa sobre o Câncer - uma instituição internacional sem fins lucrativos - . A empresa baseia-se em um modelo de inovação aberta e disfruta de parceria com empresas e institutos de pesquisa renomados, como o Instituto Butantan, a Universidade de São Paulo (USP), a Universidade Federal do Estado de São Paulo (UNIFESP), o Laboratório Nacional de Biociências (LNBio) do Centro Nacional de Pesquisas em Energia e Materiais (CNPEM) e o norte-americano Global Entrepreneurship Lab (G-Lab) do Massachussetts Institute of Technology (MIT). Em 2012, a empresa que já contava com o apoio financeiro da Financiadora de Estudos e Projetos (FINEP), foi investida por dois investidores anjos - Emílio Odebrecht e Jovelino Mineiro -, e teve 16\% de seu capital acionário adquirido pela BNDES-PAR, uma subsidiária do Banco Nacional de Desenvolvimento Econômico e Social (BNDES). Em 2013, a FINEP divulgou que a empresa receberia mais R \$ 14,7 milhões em financiamento reembolsável. Já em 2015, a empresa fechou um acordo de licenciamento de propriedade intelectual para a comercialização no Brasil e no exterior do RebmAb200, um de seus anticorpos monoclonais, com a Mersana Therapeutics, Inc, uma empresa norte-americana. Atualmente a Recepta encontra-se em fase préoperacional. A empresas possui dois produtos em fase de teste clínico (o RebmAb100 e o RebmAb600) e dois produtos em fase desenvolvimento pré-clínico (o RebmAb200 e o RebmAb700), além de quatro produtos em fase de pesquisa. (BNDES, 2012; VALOR ECONÔMICO, 2012; MERSANA, 2015 e FINEP, 2013).

\section{Considerações finais}

A interação entre universidades e empresas é aspecto relevante para a geração de inovações em indústrias baseadas em ciência, conforme referendado na literatura acadêmica sobre sistemas setoriais de inovação farmacêutica. No caso brasileiro, observa-se a participação de cinco elementos principais empresas farmacêuticas nacionais e multinacionais, empresas de biotecnologia, laboratórios públicos de pesquisa e produção de fármacos e medicamentos e universidades e centros de pesquisa - no âmbito de um sistema setorial marcado pela existência do SUS e pela participação ativa do governo como articulador do sistema de inovação em saúde.

A análise realizada neste trabalho permitiu a identificação de 243 empresas e instituições envolvidas nas atividades de fabricação de produtos farmacêuticos e farmoquímicos e condução de pesquisa e desenvolvimento científico que mantiveram relacionamentos com grupos de pesquisa cadastrados no CNPq segundo dados coletados no Censo 2010. Das 243 empresas identificadas, 42\% pertenciam ao ramo de fabricação de produtos farmacêuticos e farmoquímicos e os demais $58 \%$ estavam catalogados no ramo de P\&D científico. Os resultados apresentados apontam no sentido de uma maior intensidade nas interações com universidades por parte das empresas de $P \& D$ científico em comparação às empresas farmacêuticas e farmoquímicas.

A intensidade dos relacionamentos universidade-empresa pode ser um indicativo dos esforços de inovação empreendidos pelas empresas. Os dados revelam uma maior frequência na interação com universidades por parte das empresas de biotecnologia catalogadas sob o ramo de P\&D industrial em 
comparação com as empresas farmacêuticas e farmoquímicas. Os tipos mais frequentes de relacionamentos foram esforços conjuntos de pesquisa básica e aplicada e transferências diretas de tecnologia entre grupos de pesquisa e parceiros do setor produtivo. Apesar da importância das atividades de formação de pessoal e pesquisa básica pelas universidades, as atividades conjuntas de pesquisa aplicada e transferência de tecnologia são indicativos da atuação ativa das instituições acadêmicas brasileiras no empreendedorismo acadêmico e no fomento das inovações, conforme descrito no modelo da hélice tripla de Etzkowitz e Leydesdorf (2000).

Os dados indicam, ainda, uma elevada participação de micro, pequenas e médias empresas nos ramos de atividade selecionados. Enquanto as empresas com até 50 funcionários representam cerca de $70 \%$ e as empresas com até 500 funcionários constituíam 86\% do total de empresas, as grandes empresas com mais de 500 funcionários representavam apenas $14 \%$ da amostra. A maior proporção de grandes empresas está catalogada sob o ramo de fabricação de produtos farmacêuticos e farmoquímicos e a maior proporção de micro e pequenas empresas encontra-se no ramo de P\&D científico. Esse é um resultado esperado e coerente com a hipótese de que as grandes empresas farmacêuticas estão catalogadas sob o primeiro ramo de atividade, enquanto as novas empresas de base biotecnológica concentram-se no ramo de P\&D científico.

Outro traço marcante é a elevada concentração regional das empresas na região sudeste. Um resultado interessante é a maior dispersão das empresas de $P \& D$ científico por unidades da federação, em comparação com as empresas farmacêuticas e farmoquímicas. Esse resultado pode ser consequência da presença de 5 instituições da administração pública e 61 entidades sem fins lucrativos no ramo de atividade de $P \& D$ científico, cuja dispersão geográfica pode estar alinhada aos interesses de integração espacial do país definidos pelo Estado brasileiro.

A avaliação sobre o perfil das empresas envolvidas nas interações universidade-empresa segundo natureza jurídica e a análise exploratória da lista de empresas revelaram uma amostra de empresas e instituições ampla e diversificada que abrange todos os principais componentes do sistema setorial de inovação farmacêutica brasileira descritos na literatura acadêmica. O Refino dos dados revelou a presença de laboratórios oficias e de instituições de apoio ao desenvolvimento científico e tecnológico na lista de empresas e instituições que mantiveram relacionamentos com os grupos de pesquisa do CNPq no ano 2010, entre eles a Fundação Oswaldo Cruz e Instituto BioRio. A grande diversidade de atores integrantes do setor farmacêutico nacional parece corroborar a hipótese levantada por Cassiolato e Albuquerque (2002) de que as universidades atuam como um importante elo de convergência de informações e articulações nos sistemas de inovação em saúde humana.

Por fim, a análise exploratória das 177 entidades empresariais contidas na amostra de 243 empresas e instituições revelou que 64\% das entidades empresariais estavam voltadas à saúde humana, enquanto as demais empresas dedicavam-se à saúde animal e a atividades de P\&D científico em biocombustíveis, agropecuária e soluções ambientais. Do total de 113 empresas voltadas à saúde humana, 36 dedicam-se à produção de fitoterápicos, 5 são laboratórios oficiais, 50 são empresas farmacêuticas e 31 são empresas de biotecnologia. Há dupla contagem nesses totais porque algumas empresas produtoras de fitoterápicos também atuam na produção de medicamentos sob prescrição médicas e três laboratórios públicos constam na lista de empresas voltadas à biotecnologia, em função da atuação na pesquisa e produção de antirretrovirais e vacinas. Estima-se que 26 empresas de biotecnologia sejam constituídas sob o formato de micro e pequenas empresas nacionais de base tecnológica.

\section{Conclusões}

O estudo exploratório das empresas de fabricação de produtos farmacêuticos e farmoquímicos e $\mathrm{P} \& \mathrm{D}$ científico que mantiveram relacionamentos com grupos de pesquisa do CNPq, segundo os dados coletados no censo 2010, revelaram o nome de 243 empresas e instituições. Os tipos de relacionamento mais frequentes dessas empresas com os grupos de pesquisa envolviam a condução conjunta de pesquisas básica e aplicada e a transferência direta de tecnologia entre os atores interativos. A maioria das empresas e instituições eram micro, pequenas e médias conforme o critério de classificação por número de funcionários e encontravam-se concentradas na região sudeste do país. A filtragem dos dados revelou uma 
lista de 177 entidades empresariais, 5 instituições integrantes da administração pública e 61 entidades sem fins lucrativos. As duas últimas categorias incluem laboratórios públicos de pesquisa e instituições de apoio à infraestrutura científica e tecnológica em biociências, a exemplo dos casos da Fiocruz e do Instituto BioRio. Entre as 177 entidades empresariais, estima-se que 64\% da amostra esteja voltada a atividades voltadas à saúde humana, enquanto as demais empresas dedicam-se a condução de P\&D científico em biocombustíveis, agropecuária, soluções ambientais e produção de fármacos e medicamentos para saúde animal. O estudo logrou identificar na amostra de entidades empresariais, 50 empresas farmacêuticas e 31 empresas de biotecnologia. A diversidade de atores constituintes do sistema setorial farmacêutico brasileiro presentes na amostra selecionada age no sentido de corroborar o importante papel das universidades como elo de convergência e articulações do setor farmacêutico nacional e a atuação ativa das instituições acadêmicas brasileiras em biociências no empreendedorismo acadêmico e no fomento a inovações.

\section{Referências bibliográficas}

ABDI. Incorporação da rota brasileira biotecnológica na indústria farmacêutica brasileira: desafios e oportunidades. In: Relatório de acompanhamento setorial. Maio de 2013.

ALBUQUERQUE, E. CASSIOLATO, J. E. As especificidades do sistema de inovação do setor saúde: uma resenha da literatura como introdução a uma discussão sobre o caso brasileiro. Estudos FesSBE I, 2000.

ARAGÃO, E. Colaboração e inovação na área de biotecnologias aplicadas à saúde humana. 2011. $120 f$. Tese (Doutorado em Saúde Pública) - Instituto de Saúde Coletiva, Universidade Federal da Bahia. Salvador, 2011.

ARORA, A. GAMBARDELLA, A. The division of innovative labor in biotechnology. In: ROSEMBERG, N. et al. Sources of Medical Technology: Universities and Industry. Washington, DC: National Academy Press p.188-208, 1995

BIANCHI, C. O papel das políticas públicas no regime de inovação de biotecnologia para saúde humana. Brasil 2002-2010. 2012. 217f. Tese (Doutorado em Economia) - Instituto de Economia, Universidade Federal do Rio de Janeiro . Rio de Janeiro, 2012.

BIO-MANGUINHOS. Instituto de Tecnologia em Imunobiológicos. Disponível em: < https://www.bio.fiocruz.br/> Acesso em 2o de julho de 2016.

BIOMINAS. PWC. A indústria de biociências nacional: caminhos para o crescimento. Belo Horizonte, 2011.

BIORIO. Polo de Biotecnologia do Rio de Janeiro. Disponível em: < http://www.biorio.org.br/ > . Acesso em: Acesso em 12 de abril de 2016.

BLOOMBERG. Disponível em: <http://www.bloomberg.com/>. Acesso em 20 de junho de 2016.

BNDES. Banco Nacional de Desenvolvimento Econômico e Social. BNDES investe na Recepta Biopharma. 25 de julho de 2012. Disponível em: < http://www.bndes.gov.br/SiteBNDES/bndes/bndes_pt/Institucional/Sala_de_Imprensa/Noticias/2012/indu stria/20120725_recepta.html>. Acesso em 12 de abril de 2016.

BRASIL. Lei $\mathrm{n}^{\mathrm{o}} 12.349$ de 15 de dezembro de 2010 . Disponível em: http://www.planalto.gov.br/ccivil_03/_ato2007-2010/2010/lei/112349.htm. Acesso em: 25/07/2016.

BRASIL. Lei $\mathrm{n}^{\circ}$ 9789/1999 de 10 de fevereiro de 1999. Disponível em: < http://www.planalto.gov.br/ccivil_03/leis/L9787.htm>. Acesso em: 25 de julho de 2016.

BRASIL. Medida provisória $n^{\circ} 995$ de 19 de julho de 2010. Disponível em: <http://www.planalto.gov.br/ccivil_03/_ato2007-2010/2010/Mpv/495.htm>. Acesso em: 25/07/2016.

CEBRAP. BRBIOTEC. Brazil Biotech Map 2011. São Paulo. 2011. 
CNPq - Conselho Nacional de Desenvolvimento Científico e Tecnológico. Diretório Nacional dos Grupos de Pesquisa. Disponível em: < http://lattes.cnpq.br/web/dgp/home>. Acesso em 5 de maio de 2016.

COHEN, W. LEVINTHAL, D. A. Absorptive capacity: A new perspective on learning and innovation. In: Administrative Science Quarterly, v. 35, pp. 128-153. 1990.

CORIAT, B. ORSI, F. WEINSTEIN, O. Does Biotech Reflect a New Science-based Innovation Regime?. In: Industry \& Innovation, v.10, p. 231 - 253. 2003.

DOSI, G. MAZZUCATO, M. Knowledge Accumulation and industry evolution. The case of PharmaBiotech. Cambridge University Press, P.1-19. 2006.

ECONODATA. Disponível em: < http://www.econodata.com.br/>. Acesso em 20 de junho de 2016.

ELIASSON, G. ELIASSON, A. The Biotechnology Block of Competences. In: Revue de Economie Industrielle, v.2 , p. 7 - 26. 1996.

ETZKOWITZ, H. LEYDESDORFF, L. The dynamics of innovation: from National Systems of Innovation and Mode 2 to a Triple Helix of University-Industry-Government Relations. In: Policy Research, v.29, p. 109-23. 2000.

FINEP, 2013. Finep apoia com cerca de R\$ 15 milhões nova droga contra o câncer. Julho de 2013. Disponível em: http://www.finep.gov.br/noticias/todas-noticias/4305-finep-apoia-com-cerca-de-r-15milhoes-nova-droga-contra-o-cancer. Acesso em 1 de agosto de 2016.

FIOCRUZ. Fundação Oswaldo Cruz: uma instituição a serviço da vida. Disponível em: < http://portal.fiocruz.br/pt-br>. Acesso em 20 de julho de 2016.

FONSECA, M.G. Documento Setorial: Biotecnologia. In: BNDES, UFRJ, UNICAMP (orgs): Projeto Perspectivas do Investimento no Brasil. Rio de Janeiro. 2009.

GADELHA, C.A.G. et al. A dinâmica do sistema produtivo da saúde: inovação e complexo econômicoindustrial. Rio de Janeiro: Ed. Fiocruz, 221p. 2012.

GADELHA, C.A.G. et al. Complexo económico-industrial da saúde. GADELHA et al 2015. In: COSTA, L. BAHIA, L. GADELHA, C.A.G. org. Saúde, Desenvolvimento e Inovação. Rio de Janeiro.: CEPESC Editora v2, 2015.

GOMES, E., Clusters e biotecnologia para a superação da imitação: estudo de caso da indústria farmacêutica brasileira. 391f. Tese (Doutorado em Políticas Públicas, Estratégias e Desenvolvimento) Instituto de Economia, Universidade Federal do Rio de Janeiro. Rio de Janeiro, 2014.

GRAVAGLIA, C. et al. Entry, market structure and innovation in a "history friendly" model of the evolution of the pharmaceutical industry. In: DOSI, G. MAZZUCATO, M. (orgs). Knowledge Accumulation and industry evolution. The case of Pharma-Biotech. Cambridge University Press, P.1-19. 2006.

MCKELVEY, M. ORSENIGO, L. Pharmaceuticals as a Sectoral Innovation System. In: ESSY Project. European Sectoral Systems of Innovation. 2001.

MERSANA. Mersana Therapeutics and Recepta Biopharma S.A. Enter License Agreement for Novel Antibody. Julho de 2015. In: Press Releases \& News. Disponível em: < http://www.mersana.com/news/mersanatherapeuticsandreceptabiopharmasaenterlicenseagreementnovelan tibody>. Acesso em 12 de abril de 2016.

MOWERY, D. SAMPAT, B. Universities in National Inovations Systems”. In FAGERBERG, J. et al. (orgs). The Handbook of innovation. Oxford University press, 2005.

OECD. A framework for biotechnology statistics. 2005. 
KLEVORICK, A.K., LEVIN, R.C., NELSON R.R. e WINTER, S.G., On the sources and significance of interindustry differences in technological opportunities. In: Research Policy, 1995, v. 24, 185-205. 1995.

PARANHOS, J. HASENCLEVER, L. Alteração no padrão de esforços de inovação das grandes empresas farmacêuticas no Brasil, 2008-2011. In: XVI Congresso Latino-Ibero-americano de Gestão Tecnológica. ALTEC 2015. Porto Alegre, 2015. Disponível em: < http://www.altec2015.org/anais/altec/papers/511.pdf>. Acesso em 12 de abril de 2016.

PARANHOS, J. Interação entre empresas e instituições de ciência e tecnologia: o caso do sistema farmacêutico de inovação brasileiro. Rio de Janeiro: Eduerj. 2012.

PAVITT, K. Sectoral patterns of technical change: Towards a taxonomy and a theory. In: Research Policy v. 13, p. 343 - 372. 1984.

RECEPTA Biopharma, 2012. Disponível em: < http://www.receptabiopharma.com.br/site/pt-br/>. Acesso em 12 de abril de 2016.

REIS, C. et al. Biotecnologia para saúde no Brasil. In: BNDES Setorial, v. 32, p. 193-230 Set 2010

SEBRAE. Anuário do Trabalho na Micro e Pequena Empresa 2010/2011. São Paulo, 2011. $4^{a}$ Edição. Disponível em:<http://www.sebrae.com.br/Sebrae/Portal\%20Sebrae/Anexos/Anuario\%20do\%20Trabalho \%20Na\%20Micro\%20e\%20Pequena\%20Empresa_2010_2011.pdf>. Acesso em: 25 de julho de 2016.

SHANE, S. Academic Enterpreunership. University Spinoffs and wealth creation. Edward Elgar Publishing Limited. Massachusetts, 2004.

VALOR ECONÔMICO. BNDES e sócios privados fazem aporte de R\$ 35 milhões na Recepta Biopharma. 25 de julho de 2012. Disponível em: <http://www.valor.com.br/empresas/2763636/bndes-e-sociosprivados-fazem-aporte-de-r-35-milhoes-na-recepta-biopharma>. Acesso em 12 de abril de 2016.

VARGAS, M. A.; BRITTO, J. A systemic innovation policy with an inclusive perspective: the evolution of the Brazilian policy to the pharmaceutical sector. In: 3th Globelics International Conference, 2015, Havana, Cuba. 3th Globelics International Conference: Innovation to reduce poverty and inequalities for Inclusive and sustainable development, 2015.

VARGAS, M. A.; GADELHA, CAG.; MALDONADO, JMSV.; COSTA, L. Innovation in pharmaceutical and health biotechnology industries: challenges for a virtuous agenda. In: Revista de Saúde Pública, v. 46. p. 37-40. 2012.

VARGAS, M. et al. Indústria de base química e biotecnologia voltadas para a saúde no Brasil: panorama atual e perspectiva para 2030. In: Fiocruz; Ipea, Ministério da Saúde; Secretaria de Assuntos Estratégicos da Presidência da República. (Org.). A saúde no Brasil em 2030: prospecção estratégica do sistema de saúde brasileiro: desenvolvimento produtivo e complexo da saúde. 22 ed. Rio de Janeiro: Editora Fiocruz, v. 05, p. 29-78. 2013. 